\title{
Les approvisionnements morutiers de la Guadeloupe durant la Seconde Guerre mondiale
}

\section{Myriam Alamkan}

During the Second World War, isolated in the absence of commercial relations with France, Guadeloupe used the peculiarities of its status as a French colony to continue to provide its population with essential cod from Saint-Pierre and Miquelon and Newfoundland and Labrador without its own commercial fleet. Although officially linked to Vichy France, Guadeloupe took advantage of the improbable agreements signed with the United States and, when administered by Free France, it collaborated with Newfoundland. To alleviate the shortage, the Guadeloupe authorities for the first time promoted the consumption of fresh fish as a substitute for cod.

Entre 1635 et 1946, la Guadeloupe est, généralement, une colonie française. En temps de guerre, la question de l'approvisionnement a été une préoccupation puisque les conflits précédents ont bien souvent entraîné des restrictions pour la population. C'est dans le strict cadre légal imposé par la France que la colonie doit faire face à l'ouverture du conflit en 1939. Aucun gouverneur de la Guadeloupe ${ }^{1}$ n'avait eu à se prononcer sur la légitimité des ordres reçus depuis la France entre collaboration et résistance à l'occupation.

\footnotetext{
1 Le titre officiel est gouverneur de la Guadeloupe et dépendances. Nous avons conservé une version réduite du titre officiel dans ce texte pour faciliter la lecture.
}

The Northern Mariner / Le marin du nord, XXIX, No. 4 (L'hiver 2019), 335-358 


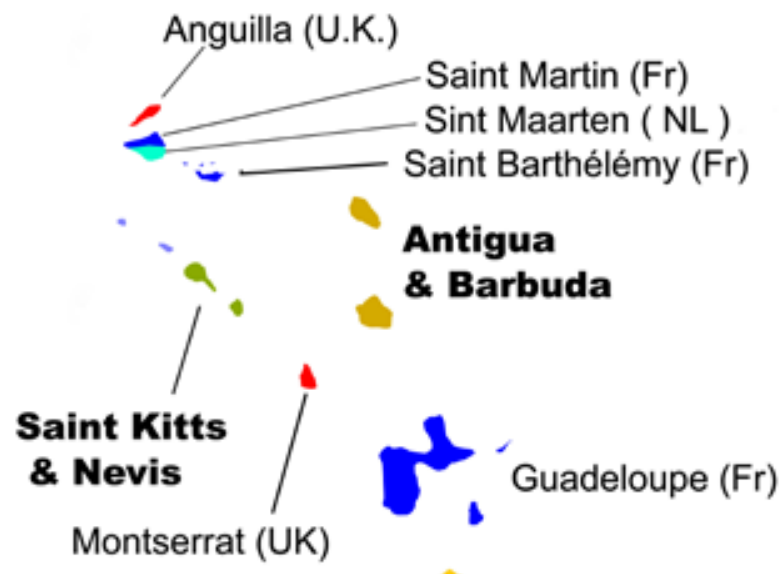

\section{Dominica}

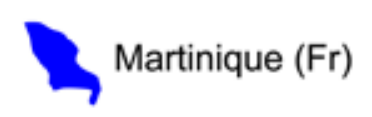

Source:

Wikimedia

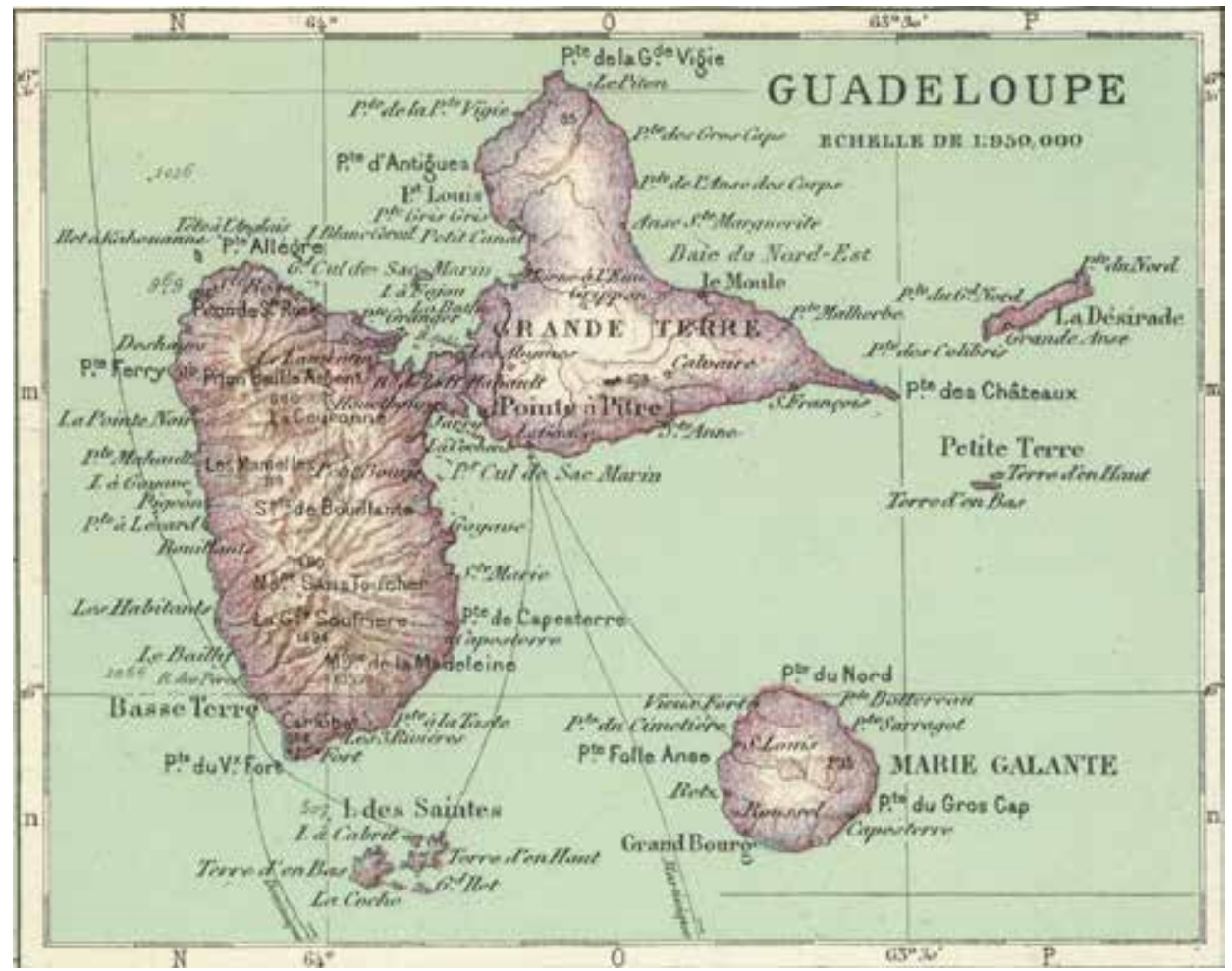

Détail des Antilles. Carte 69 dans Vivien de Saint-Martin \& Schrader, Atlas Universel de Géographie (Librairie Hachette, 1931). 
Constant Sorin $^{2}$, gouverneur de la Guadeloupe, choisira rapidement le camp du maréchal Pétain et la Guadeloupe sera collaborationniste après l'armistice du 22 juin 1940. Or les décisions politiques françaises vont avoir un impact sur les approvisionnements de ses colonies et particulièrement pour la Guadeloupe. Comment se ravitailler durant le conflit?

C'est ce que nous nous proposons de montrer à travers l'exemple de la morue. Nous nous intéresserons d'abord à établir comment la morue est devenue une ressource alimentaire incontournable de la Guadeloupe. Puis, nous examinerons les solutions d'approvisionnement portées par le régime de Vichy localement. Et enfin les solutions proposées par l'administration de la France libre pour se procurer de la morue à Saint-Pierre et Miquelon, ou à Terre-Neuve et au Labrador. Ce sont ces dispositifs économiques particuliers à la Guadeloupe, dus à la situation de guerre en Europe, que nous nous proposons de découvrir à travers cette étude.

\section{La morue, ressource alimentaire incontournable}

La loi du 11 juillet 1938 sur l'organisation générale de la nation pour le temps de guerre $^{3}$ prépare le cadre législatif et consacre son titre IV à l'aspect économique du conflit. Or la législation nationale doit être adaptée pour être applicable aux colonies. C'est chose faite par l'adoption du décret du 2 mai $1939^{4}$ qui élargit son application aux territoires d'outre-mer, donc à la Guadeloupe. Cependant, la Guadeloupe est composée de trente-deux communes dont deux disposent d'un régime fiscal particulier.

\section{Présentation de la Guadeloupe à l'ouverture du conflit}

Au 2 mai 1939, la Guadeloupe est composée de sept îles principales habitées : la Grande-Terre, Basse-Terre, Terre-de-Haut, Terre-de-Bas, MarieGalante, la Désirade, Saint-Barthélemy et la partie française de Saint-Martin. Administrativement, les îles sont subdivisées en communes administrées par des maires soumis au pouvoir du gouverneur ${ }^{5}$ représentant le gouvernement central. Saint-Barthélemy et la partie française de Saint-Martin sont ordinairement désignées comme étant les «îles du Nord », tant dans la langue courante que dans les divers documents administratifs. Les îles du Nord ont un statut particulier du fait de leurs

\footnotetext{
2 SORIN, Constant, Louis, Sylvain. Né le 27/07/1901 à Landerneau, Finistère. Décédé le 20/01/1970 à Neuilly, Haut de Seine. Militaire, diplômé de Saint-Cyr. En 1936 il devient inspecteur des colonies et démissionne de l'armée. Chevalier de la Légion d'honneur le 15 juillet 1939 suite à ses missions en Afrique occidentale et en Afrique équatoriale. Il est gouverneur de la Guadeloupe du 30 avril 1940 à juillet 1943. Base LEONORE de la Légion d'honneur. Côte 19800035/1033/19221.

3 Journal Officiel de la République Française (J.O.R.F) du 13 juillet 1938, page 8330.

$4 \quad$ J.O.R.F du 7 mai 1939, page 5814.

5 SEMPAIRE, Élaine. La Guadeloupe an Tan Sorin (1940-1943). Ibis Rouge éditions, 2004. L'auteur énumère les pouvoirs des gouverneurs : promulgation des textes élaborés par le pouvoir central, pouvoir militaire, politique restreinte, pouvoir judiciaire, pouvoir de direction de services administratif et de police, pages 22-23.
} 
histoires coloniales respectives (ex-colonie suédoise pour Saint-Barthélemy et île binationale ${ }^{6}$ pour Saint-Martin). Leur éloignement, près de 300 kilomètres, du reste de la Guadeloupe leur concède un statut fiscal différent. Le port principal de SaintBarthélemy, Gustavia, et celui de Saint-Martin, Marigot, sont tous deux des ports francs, exempts de droits de douane. Ainsi les marchandises en provenance des îles du Nord sont considérées en Guadeloupe comme des marchandises d'importation.

Ainsi, l'arrêté $n^{\circ} 54$ du 8 janvier $1940^{7}$ signé par le gouverneur de la Guadeloupe, pris pour réglementer la « vente et la circulation des denrées, produits, matières et objets de première nécessité » exclut Saint-Barthélemy et Saint-Martin de son champ d'application. Cette exception pour les îles du Nord permet aux habitants de commercer librement avec la Guadeloupe mais également de continuer à faire leur commerce habituel avec les îles anglaises voisines, loin des yeux du gouverneur. Examinons ce que l'administration considère comme produits de première nécessité pour la Guadeloupe et pourquoi la morue en fait partie.

\section{La morue est désignée produit de première nécessité}

Nous constatons à la lecture du titre 1 de l'article 1 de l'arrêté du 8 janvier 1940 que la morue est l'unique poisson concerné : «denrées périssables ou susceptibles de gros déchets : farine de froment, pomme de terre, oignon, aulx, viandes en saumure (bœufs, porcs), morue, graisse alimentaire, sauce rouge dite beurre rouge, beurre frais, fromage, pâtes alimentaires. » Cette liste est complétée dans le même article de l'arrêté relatif aux denrées non périssables : « huiles de cuisine (arachide, coprah, coton, maïs, etc.), huiles de table (olive et autres coupages), beurre de table (en boîtes métalliques ou en verre), saindoux, riz blanc et jaune toutes provenances, savon de ménage, haricots, pois, lentilles, vin blanc ou rouge (importés en barriques), rhum, sucre, lait en boîtes (sucré ou non), corned beef, sardines, maïs, sel, pétrole, essence, huile de graissage » (sic). Si aucun poisson frais ne fait partie de la liste des denrées de première nécessité, comment la morue s'est-elle imposée dans le régime alimentaire en Guadeloupe?

Elle s'est imposée dans le régime alimentaire des Guadeloupéens durant la période esclavagiste ${ }^{8}$. Alors que les eaux guadeloupéennes sont riches en poissons, la politique coloniale a privilégié la morue et d'autres poissons issus des mers tempérées dans l'alimentation des Guadeloupéens. La morue est présente dans les rations obligatoires fournies par le maître à ses esclaves et cette obligation est inscrite dans l'article 22 du Code Noir publié en 1685. Depuis, l'administration encourage sa consommation et poursuit cette politique après la seconde abolition

\footnotetext{
6 Cette dernière partage son territoire avec la colonie néerlandaise Sint-Maarten.

7 Archives Départementale de la Guadeloupe (ADG). Journal Officiel de la Guadeloupe (JOG) du 11 janvier $1940.3 \mathrm{~K} 132$.

8 ALAMKAN, Myriam. La tropicalisation de la consommation de la morue : l'exemple de la Guadeloupe. Acte des Quatrièmes journées d'histoire de la Grande Pêche (30-31 octobre 2009). Édité par la Société d'archéologie et d'histoire de la Manche, 2012.
} 
de l'esclavage de $1848^{9}$.

La morue s'est imposée au fil des siècles comme une ressource essentielle pour la Guadeloupe. En effet, le poisson frais peut voir ses chairs altérer et provoquer des intoxications alimentaires parfois fatales. En $1940^{10}$, trois modes de conservation sont utilisés en Guadeloupe de manière inégale : le froid, le salage et le fumage. Seule Saint-Barthélemy dispose d'une organisation dévolue au salage des poissons locaux. Saint-Martin possède une conserverie. Et en Guadeloupe, les ports principaux de Basse-Terre et de Pointe-à-Pitre se dotent à cette époque de « deux entrepôts frigorifiques, qui permettront le ravitaillement des populations urbaines, en attendant 1 'achat de camionnettes glacières ${ }^{11}$. » Or l'activité de pêche ne se limite pas aux seuls ports de Basse-Terre et de Pointe-à-Pitre. Quasiment toutes les communes de la Guadeloupe accueillent une activité de pêche. Cependant, à mesure que l'on s'éloigne du bord de mer, l'altération des chairs des poissons frais est toujours plus rapide que celle de la morue salée qui dans ce milieu tropical humide finit elle aussi par se corrompre.

C'est grâce à ses qualités de conservation que la morue est vendue dans les boutiques des usines de cannes à sucre, ou les « lolos » (épiceries traditionnelles de la Guadeloupe) où elle est proposée sous forme de portion de demi-quart de livre ou portion de 62,5 grammes. Ce qui permet d'entretenir l'illusion d'un repas avec « chair » ${ }^{12}$ synonyme d'un vrai repas pour les Guadeloupéens. Elle est préférée au poisson frais local dont les approvisionnements sont moins réguliers. En outre il existe des risques réels d'intoxications alimentaires dues à la ciguatéra ${ }^{13}$ ou à l'ingestion de poissons venimeux ${ }^{14}$.

Si la morue apparaît dans la cuisine guadeloupéenne, sa qualité est très aléatoire. Voilà ce qu'en disait le gendarme Georges Bonnemaison qui a exercé en Guadeloupe entre 1900 et $1903^{15}$ : « Le poisson est assez commun quoiqu'assez cher à la Pointe-à-Pitre, mais comme il vient des environs, surtout de MarieGalante et des Saintes, il n'est pas toujours frais. Il est abondant dans la rade, mais il est souvent venimeux et on ne permet pas de l'y pêcher. La morue est chère et pas toujours bonne. »

Malgré ces problèmes, la morue est un ingrédient incontournable de la cuisine

9 BOUTIN, Raymond. Vivre ensemble en Guadeloupe 1848-1946 : un siècle de construction. Ibis Rouge Éditions, 2009. Page 103.

10 BLANCHE, Lénis. La pêche maritime en Guadeloupe. ADG INC 940, pages 21 à 25.

11 Id. page 22.

12 BOUTIN, Raymond. Vivre ensemble en Guadeloupe, 1848-1946 : un siècle de construction. Ibis Rouge Éditions, Matoury, Guyane, 2009.

13 La ciguatéra est causée par l'ingestion des poissons contaminés par une algue microscopique : Gambierdiscus toxicus. Lire également l'arrêté préfectoral n 2002-1249 (Préfecture de Guadeloupe) relatif à l'interdiction à la vente et à la consommation de poissons dangereux.

14 ALAMKAN, Myriam. La tropicalisation de la consommation de la morue : l'exemple de la Guadeloupe. Acte des Quatrièmes journées d'histoire de la Grande Pêche (30-31 octobre 2009). Édité par la Société d'archéologie et d'histoire de la Manche, 2012.

15 BONNEMAISON, Georges. La Guadeloupe en zigzag. Présenté par René Martin. Edition Caret, 2001. Page 59. 
et c'est avec l'arrêté 1970 du 28 décembre 1940 que le gouverneur de la Guadeloupe établit un monopole d'importation de la morue française ou étrangère qu'il réserve au service du Ravitaillement.

\section{L'économie de la Guadeloupe avant l'ouverture du conflit}

L'essentiel du commerce de la colonie transite par ses deux ports principaux: Pointe-à-Pitre et Basse-Terre. L'économie de la Guadeloupe, hors îles du Nord, est largement basée sur les cultures d'exportation de la banane et de la canne à sucre $^{16}$. Nous rappelons ici que les aliments de première nécessité énumérés par l'administration coloniale sont rarement produits localement. Cela est dû à la nature même de l'économie coloniale qui fait des colonies des "marchés pour la production française; il est interdit aux colonies de développer, de fabriquer les produits que la France peut leur fournir, de les acheter à plus bas prix à l'étrange ${ }^{17}{ }^{»}$. Ainsi la Guadeloupe importe essentiellement de France de la viande et des conserves de viande, des poissons secs, fumés ou salés, de la farine de froment et des pommes de terre.

Cependant, les importations reposent majoritairement sur des dessertes régulières entre la France et la Guadeloupe. Examinons la réalité de ces dessertes pour le port de Pointe-à-Pitre en 1938. Il était approvisionné à partir de la France, des États-Unis et du Canada ${ }^{18}$ par la Compagnie Générale Transatlantique par ses lignes France-colonie (bimensuelle), France-Haïti (mensuelle); les Messageries Maritimes : la ligne Marseille-Nouméa (tous les 45 jours) et la Société Générale des Transports Maritimes à Vapeur (sans horaire fixe). Il existe aussi des lignes internationales se résumant aux rotations anglaises de la Dess Bermuda Line de New York à Trinidad (fréquence bimensuelle); Canadian National Steamships du Canada au Venezuela (fréquence mensuelle) et états-uniennes par l'American Caribbean Line de New York à Demerara (fréquence bimensuelle); l'Ocean Dominion Steamship Corporation (même ligne, même fréquence) et l'Aluminium Line de la Nouvelle-Orléans à Demerara. La colonie ne dispose pas de flotte commerciale pouvant remplacer ces liaisons à court, moyen ou long terme. De fait, la Guadeloupe a besoin de maintenir de bonnes relations commerciales maritimes avec le Royaume-Uni de Grande-Bretagne et d'Irlande du Nord et les États-Unis d'Amérique pour maintenir ses approvisionnements ordinaires, en sus de la France.

Avant le début du conflit, la Guadeloupe importait annuellement pour 5 millions de francs de poissons dont $98 \%$ de ses marchandises étaient introduites par des armements français. Elle importait de la morue salée et séchée, du poisson fumé comme le hareng, des poissons préparés (sardine à l'huile, maquereau au

\footnotetext{
16 SEMPAIRE, Élaine. La Guadeloupe an Tan Sorin (1940-1943). Ibis Rouge éditions, 2004. Pages 80 à 124.

17 Id. page 81.

18 ADG. Rapport Coursin sur l'appontement de la Basse-Terre. Fond de l'Incendie de 1955 (INC) 940. Série M : administration générale de la colonie 1926-1948.
} 
vin blanc $\left.\ldots{ }^{19}\right)$. À cause de la déclaration de guerre, la Guadeloupe ne peut que se tourner vers un approvisionnement de proximité : Saint-Pierre et Miquelon, les États-Unis et le Canada.

Or cette réorientation du commerce se heurte à plusieurs difficultés que subit le gouverneur Sorin. La Guadeloupe n'est pas concernée par le décret national du $1^{\text {er }}$ septembre 1939, prohibant le commerce étrange ${ }^{20}$. En théorie, toutes ses lignes commerciales sont maintenues. Cependant, au 29 mai $1940^{21}$, la Guadeloupe n'a plus beaucoup de relations directes : « les relations sont fréquentes dans le sens Martinique Guadeloupe, plus rares dans le sens Guadeloupe Martinique. » Elles sont rares avec la Guyane. Cette destination est desservie par un unique navire qui assure également des escales dans les îles du Vent (Sainte-Lucie, Barbade, SaintVincent, Grenade et Trinidad), la Guyane anglaise et Demerara. Les liaisons avec les îles Sous-le-Vent sont faites par des cargos américains de façon irrégulière. De plus, la desserte aérienne de la Guadeloupe est balbutiante. Les hydravions ne peuvent servir au ravitaillement de la colonie.

Pour acheter à l'étranger, il faut pouvoir disposer de devises. La Guadeloupe disposait d'environ 100000 dollars US ${ }^{22}$ qu'il faut affecter « à la satisfaction des besoins essentiels. » La question primordiale est à qui acheter la morue nécessaire à la population locale, sans favoriser la fuite de capitaux essentiels à la survie de l'archipel guadeloupéen. La réponse la plus évidente pour les autorités est SaintPierre et Miquelon.

\section{Les approvisionnements en morue durant l'administration de Vichy : Saint- Pierre et Miquelon fournisseur principal}

Dans la mémoire de beaucoup de Guadeloupéens, l'administration Sorin ou «Tan Sorin » (littéralement, l'époque de Sorin) est synonyme de la période de la Seconde Guerre mondiale. Le gouverneur Sorin a connu quelques hésitations après la capitulation française de 1940. Il a choisi d'être fidèle au régime de Vichy' ${ }^{23}$, entraînant avec lui l'ensemble de la colonie de la Guadeloupe malgré l'existence d'une opposition politique locale ${ }^{24}$ encline à continuer le combat.

Pour l'aspect militaire, le commandement de la zone est placé dans les mains de l'amiral Robert ${ }^{25}$ qui est nommé au poste de Haut-Commissaire pour l'Atlantique

\footnotetext{
19 Id. La pêche maritime à la Guadeloupe, décembre 1940.

20 Id. Lettre de l'amiral Robert au ministre des Colonies.

21 Id. Lettre du gouverneur de la Guadeloupe du 29 mai 1940.

22 Id. Lettre de l'amiral Robert au ministre des colonies, 14 mars 1940.

23 Voir Dr Schneyder, La Seconde Guerre mondiale, dans Élisabeth Antébi. Histoire des Antilles et de la Guyane, ouvrage collectif, Éditions Arawak, Fort-de-France 1978, p. 287.

24 SEMPAIRE, Elaine. La Guadeloupe an Tan Sorin (1940-1943). Éditions Ibis Rouge, 2004. Pages 34 à 41 .

25 ROBERT, Georges, Achille, Marie, Joseph. Né le 31 janvier 1875 à Courseulles, Calvados. Décédé le 2 mars 1965, à Paris, Ile-de-France. Il est nommé commandant en chef des Forces Maritimes de l'Atlantique Ouest le 2 septembre 1932 avec autorité sur les Antilles et la Guyane française et Saint-Pierre et Miquelon. Puis il reçoit le titre de Haut-Commissaire de la République aux Antilles
} 
Ouest. Il se rallie au maréchal Pétain après l'armistice de juin 1940. En réaction, la Grande-Bretagne décide de placer ces îles en état de blocus maritime en août 1940. Or il n'allait pas être des plus hermétiques à cause du rôle des États-Unis d'Amérique qui vont briser le blocus anglais en devenant fournisseur pour la Martinique et la Guadeloupe.

\section{Une administration sous l'influence de l'amiral Robert, Haut-Commissaire de la République aux Antilles et en Guyane française}

Les gouverneurs de la Guadeloupe et de la Martinique ont tous les mêmes prérogatives. Le rôle de l'amiral Robert aurait dû se concentrer uniquement sur l'organisation militaire de la zone dont il est officiellement chargé. Dans une lettre adressée au ministre des Colonies ${ }^{26}$ le 14 mars 1940 , voilà comment il justifie son intervention dans le domaine civil : « J'ai été amené à différentes reprises à m'intéresser à la vie économique des Colonies dont la protection m'a été confiée en raison des incidences que les difficultés du ravitaillement, ou, plus simplement la gêne du commerce et les difficultés monétaires pouvaient y avoir sur l'ordre public. » L'amiral Robert va exercer une réelle tutelle sur les gouverneurs de la zone. En Martinique, l'expression « tan Wobè » (littéralement l'époque de Robert), synonyme de la Seconde Guerre Mondiale, montre bien l'ascendant de ce dernier sur le gouverneur de la Martinique, Yves-Marie Nicol. L'amiral Robert se charge alors d'organiser le ravitaillement des colonies, en sus de ses préoccupations militaires.

Il a sous son commandement une partie de la flotte française qui a rejoint les Antilles après la défaite de 1940 dont le croiseur-école Jeanne d'Arc, le Barfleur, le porte-avion Béarn ainsi que le croiseur Émile Bertin. L'amiral Robert envoie en Guadeloupe le croiseur-école Jeanne d'Arc. Mais il n'y a pas d'attaque allemande, ni avant l'armistice ni après. Et l'armée française basée en Martinique et en Guadeloupe n'organise aucune attaque contre l'ennemi. Pire, en Guadeloupe les marins de la Marine Nationale s'en prennent à la population civile ${ }^{27}$ et bien longtemps après la fin du conflit, le souvenir des marins du Jeanne d'Arc sera synonyme de terreur pour les Guadeloupéens de couleur noire. Sans oublier que l'arrivée de l'Émile Bertin et de l'or de la Banque de France à la Martinique est une très grande préoccupation pour les autorités britanniques.

Élaine Sempaire ${ }^{28}$ explique ainsi les premiers jours de la mise en état de blocus

\footnotetext{
et en Guyane françaises à partir du 14 septembre 1939. Après son départ de la Martinique, suite au ralliement de la colonie à la France libre, il poursuit son activité au sein de la marine de Vichy du 14 juillet 1943 au $1^{\text {er }}$ septembre 1944. Condamné par la Haute Cour de Justice le 14 mars 1947 à 10 ans de travaux forcés et à la dégradation nationale à vie pour ses activités en Martinique. Amnistié par le Président du Conseil le 15 avril 1954 après avoir obtenu une remise totale de peine pour les travaux forcés le 2 juillet 1947. (Extrait de la base LEONORE de la Légion d'honneur. Côte 19800035/659/75842).

26 ADG. INC 135.

27 SEMPAIRE, Élaine. La dissidence an Tan Sorin. Éditions Jasor, page 35.

28 La Guadeloupe an Tan Sorin (1940-1943). Ibis Rouge éditions, 2004.
} 
de la Martinique et de la Guadeloupe. Le 28 juin 1940, Sir Young, gouverneur général de la Trinité et des Antilles anglaises, se rend à la Martinique. Selon les dires de l'amiral Robert, le but de cette visite est d'organiser une prise de contrôle de la colonie par le Royaume-Uni. Le 29 juin 1940, le croiseur britannique Fidji mouille en rade de Fort-de-France, chef-lieu de la Martinique. L'attitude de l'amiral change à la suite de l'attaque anglaise sur la flotte française à Mers el-Kébir le 3 juillet : " l'amiral Robert convoque le commandant du Fidji et l'invite à quitter dans les plus brefs délais les eaux territoriales de la Martinique ${ }^{29}$.» Les colonies de Guadeloupe et de Martinique sont donc en état de blocus par les forces anglaises qui arraisonnent des bâtiments de commerce dans les parages des deux colonies. Élaine Sempaire poursuit en expliquant que " toute unité navale ou aérienne quittant l'île [la Martinique] est arrêtée ou détruite. Toutes les importations de vivres sont arrêtées. Le courrier est arrêté avant son arrivée. Le câble qui relie la Martinique à Saint-Thomas est cisaillé. Un blocus aussi rigoureux isole également la Guadeloupe ${ }^{30} \gg$.

Cependant, l'amiral Robert va mener des négociations avec les Anglais et les Américains pour assurer le ravitaillement. C'est donc vers lui que vont transiter les informations liées au blocus. C'est également lui qui signera des accords avec les Américains qui ont accepté de devenir fournisseurs des colonies françaises tout en obtenant leur désarmement.

Les accords Robert-Greenslade ${ }^{31}$ masquent l'américanophobie des militaires français basés aux Antilles qui confondent bien souvent les États-Uniens avec l'ennemi. Une Guadeloupéenne, madame Andrézine Col, alors enfant, nous a confié qu'on leur avait fait réaliser à l'école des caches pour les lampes et que le gouvernement avait demandé de peindre les toitures en vert contre les attaques aériennes. Et elle concluait que personne n'avait pu peindre ses toitures faute de peinture disponible à la vente. Il n'y a pas eu d'attaque aérienne en Guadeloupe mais Jean-Baptiste Bruneau ${ }^{32}$ note que des survols américains de la colonie ont bien eu lieu, contribuant ainsi à la paranoïa ambiante.

Comment la Guadeloupe est-elle parvenue à briser efficacement le blocus anglais? Ce n'est pas la première fois que l'archipel est soumis à un blocus et par le passé ils ont connu de grand succès. Or la Guadeloupe de 1940 ne dispose pas d'une flotte offensive. Si ce n'est pas les forces navales françaises qui ont brisé le blocus, il faut le mettre au crédit des négociations menées entre l'administration de Robert et les États-Unis d'Amérique.

\section{Organisation d'une filière d'achat à Saint-Pierre et Miquelon}

L'archipel français de l'Atlantique Nord, Saint-Pierre et Miquelon, est le seul capable de ravitailler la Guadeloupe en morue française mais leur commerce subit aussi les effets de la guerre. À partir de l'armistice de 1940, Saint-Pierre et Miquelon comme les Antilles et la Guyane sont dirigés par des fidèles au maréchal Pétain chapeauté par l'amiral Robert en charge de la défense militaire.

Toutes les colonies françaises de l'Atlantique ne peuvent plus compter sur 
la France pour s'approvisionner. Or ces colonies sont géographiquement voisines des alliés historiques de la France : le Canada, les colonies britanniques et enfin les États-Unis d'Amérique. Comme dans les Antilles, l'administrateur de SaintPierre et Miquelon a lui aussi des problèmes d'approvisionnement. C'est ainsi qu'il prendra contact avec le consul général britannique à New York et avec l'attaché commercial français à Washington et Ottawa ${ }^{33}$. C'est auprès des autorités canadiennes que se négociera le futur des 12 chalutiers, chargés de poissons, piégés par la guerre à Saint-Pierre. Les autorités canadiennes vont accepter que certains d'entre eux puissent partir vers la Martinique.

Bien que l'autorisation donnée par les Canadiens ne concerne que la Martinique, nous trouvons la trace de plusieurs chargements de morue de Saint-Pierre introduits en Guadeloupe après l'armistice de juin 1940 sans avoir la preuve formelle que ce soit à partir des 12 chalutiers précédemment cités. L'autorisation a-t-elle été élargie à d'autres chalutiers saint-pierrais? Cependant, la Guadeloupe a importé de la morue de Saint-Pierre et Miquelon après l'armistice. Ces importations ont été faites par l'administration de la Guadeloupe conjointement avec la Martinique ${ }^{34}$.

Dans une lettre datée du 31 juillet 1940, l'amiral Robert écrit au gouverneur de la Guadeloupe : «Le 28 juillet, le chalutier Le Volontaire a quitté Saint-Pierre et Miquelon pour la Guadeloupe et la Martinique avec 250 tonnes de morues vertes pour chacune des deux colonies. Cette quantité pourrait vous paraître forte en ce qui concerne la Guadeloupe. Il importe cependant de tenir compte d'une consommation assez forte de poisson frais et de la possibilité d'en effectuer la salaison si nécessaire, le prix de cette denrée, en francs est en effet extrêmement intéressant.

L'administrateur de Saint-Pierre et Miquelon a réquisitionné et mis en séchage la morue nécessaire à nos besoins sur les bases de 250 tonnes par Colonie - arrivage mi-septembre à 1100 francs les 200 kilos, fret 1000 francs les 1000 kilos - Le tout payable en francs ${ }^{35}$.»

Lorsque le gouverneur de la Guadeloupe souhaite faire un achat quelconque, il faut qu'il se soumette à la procédure administrative ordinaire pour qu'un titre de paiement soit émis puis payé par les services financiers de la colonie. Son plus haut représentant est le trésorier-payeur de la Guadeloupe, qui relève du ministère des Finances. Ce haut fonctionnaire réunit en ses mains les fonctions d'encaissement des recettes et de chargé des dépenses. C'est en quelque sorte le «banquier» de la colonie. Voici un exemple de la procédure mise en place par le trésorier-payeur de la Guadeloupe ${ }^{36}$ pour l'achat de morue par l'intermédiaire de la maison Robin de

33 Rapport mis en ligne par http://www.grandcolombier.com/histoire/ Paragraphe 23, page 22. Report $n^{\circ} 79$ Historical section (GS) Army Headquarters 30 Jan. 59. Saint-Pierre and Miquelon during the Second World War. Paragraphe 23, page 22.

34 ADG. INC 135. Lettre du trésorier-payeur de la Guadeloupe au gouverneur de la Guadeloupe. Série M.

35 Id.

36 Id. Lettre du trésorier-payeur de la Guadeloupe au gouverneur de la Guadeloupe. 
Saint-Pierre et Miquelon : « le Trésor de Saint-Pierre qui me donne préalablement son accord, paye le vendeur. Je reçois le prix de l'acquéreur et je rembourse SaintPierre par un mandat sur le Trésor. L'opération que j'ai déjà réalisée accorde des facilités au commerce sans présenter d'inconvénients; de plus, elle est intéressante pour le Trésor local puisqu'elle augmente le volume de mon compte courant. »

Si le mécanisme de paiement présente des avantages pour les autorités guadeloupéennes, elle conduit à un problème de réactivité du trésorier-payeur face aux augmentations du prix de la morue. En effet, une première cargaison a été introduite en Guadeloupe au prix de 6,75 frs/kilo. Mais le prix passe rapidement à 10 frs/kilo en novembre 1940. Cette augmentation est supérieure à celle ${ }^{37}$ observée par le trésorier-payeur de la Guadeloupe pour les achats réalisés par le commerce local. Le prix de la tonne passe dans ce cas de 7000 frs à 7500 frs la tonne alors que la morue achetée par voie administrative revient à 10960 frs/tonne.

La morue introduite par voie administrative provient en partie de la réquisition faite par l'administrateur de Saint-Pierre et Miquelon et plus tardivement par des achats faits par la Mission d'Achat aux États-Unis. Examinons d'abord le mécanisme des achats à Saint-Pierre et Miquelon. En août 1940, l'administrateur a réquisitionné environ 4300 tonnes de morues vertes pour les Antilles. Un premier lot de 1500 tonnes a été séché et représente désormais un lot de 1050 tonnes dont 530 tonnes sont expédiées dès le 26 octobre $^{38}$. C'est à partir de cette expédition que les problèmes de prix de la morue opposent les deux colonies. Le trésorierpayeur de la Guadeloupe n'hésite pas à demander au gouverneur de l'archipel de faire appel à la «solidarité intercoloniale » pour réduire la pression sur le Trésor de la Guadeloupe en intervenant sur plusieurs leviers : permettre que le commerce local puisse acquérir une partie de la morue réquisitionnée et diminuer les achats de morue par l'intermédiaire du programme de ravitaillement en poisson frais. En fait, le trésorier souhaite que la colonie n'importe plus que 50 à 75 tonnes par mois au maximum par la voie administrative.

Les présidents des chambres de commerce de Basse-Terre et de Pointe-à-Pitre estiment que le prix de détail de la morue achetée par la voie administrative à la somme de 10960 frs la tonne se traduirait pour la population à des prix de détail entre $13,50 \mathrm{frs} / \mathrm{kg}$ et 14,60 frs $/ \mathrm{kg}$; ainsi, deux expéditions de 32 tonnes puis de 73 tonnes de morue introduites par le commerce local approchent pour la même période les $8 \mathrm{frs} / \mathrm{kg}$ au prix de gros. L'estimation du prix de gros de la morue achetée par voie administrative la porterait à $12,65 \mathrm{frs}^{39} / \mathrm{kg}$. Le gouverneur Constant Sorin tranche le problème : la Guadeloupe payera au nom de la « solidarité nationale et coloniale que la Guadeloupe contribue à assurer un débouché à la seule exportation

\footnotetext{
37 Id.

38 Id. Télégramme de l'administrateur de Saint-Pierre et Miquelon au Haut-commissaire Robert du 8 novembre 1940.

39 Id. Lettre du président de la Chambre de commerce de Basse-Terre du 21 novembre 1940 et lettre du président de la Chambre de commerce de Pointe-à-Pitre du 15 novembre 1940 au gouverneur de la Guadeloupe et dépendances.
} 
de Saint- Pierre et Miquelon ${ }^{40}$. » Cependant il limite ses importations à 75 tonnes/ mois, soit 200 tonnes pour le trimestre, et ne souhaite «pas faire exécuter les dernières réquisitions portant sur 2800 tonnes de morue verte. » Or le plan de ravitaillement pour le premier trimestre de 1941 pour la colonie de la Guadeloupe fixe les besoins de l'archipel à 400 tonnes/mois pour une valeur de 2200000 frs et indique Saint-Pierre et Miquelon comme l'unique provenance.

Au premier trimestre de 1941, l'approvisionnement de la Guadeloupe dépend de sa production locale, des importations françaises des colonies d'Afrique du Nord et des importations de l'Amérique. Durant la visite de l'amiral Robert en Guadeloupe le 28 avril 1941, le Haut-Commissaire aux Antilles et à la Guyane reçoit en audience particulière la Chambre de Commerce ${ }^{41}$ et parmi les différents points abordés figure l'approvisionnement en morue. Au mois de mai 1941, l'administration avait affecté 10000 dollars US à l'achat de salaisons d'importation. Mais la Chambre de Commerce souhaitait réserver l'ensemble de ces devises à l'achat de morue exclusivement dans une lettre du 24 avril, puis avait répété ses propos devant l'amiral Robert : «La morue, véritable aliment du pauvre ici, constitue la base essentielle de l'alimentation des campagnes. Cette marchandise, en effet, qui se conserve, est achetée par les campagnards en quantité suffisante pour plusieurs jours quand ils se rendent au bourg voisin. À ce titre, elle ne saurait être remplacée ici par des salaisons d'importation ni par le poisson pêché ici. Elle est d'autre part meilleur marché que les viandes salées importées, répond d'avantage au goût des consommateurs, et a une valeur nutritive indiscutablement supérieure. » L'administration de la Guadeloupe finira d'ailleurs par suivre ces recommandations. Mais une réserve en dollars pour faire des achats signifie donc qu'il s'agit de morue étrangère achetée par voie administrative. Examinons maintenant cette procédure d'achat qui est quelque peu différente de celle utilisée avec Saint-Pierre et Miquelon.

Plusieurs télégrammes officiels évoquent l'existence de la Mission d'Achat agissant pour le compte de la colonie aux États-Unis ${ }^{42}$. Et dès le 6 novembre $1940^{43}$, le gouverneur Sorin analyse les bienfaits de la création d'un organisme officiel français à New York : «Au vu d'un plan de ravitaillement et de devise adressé au Ministre des Colonies, un crédit global de dollars serait accordé à l'Attaché financier à New York. L'Office des Changes local ne s'adresserait plus en France

\footnotetext{
40 Id. Lettre du gouverneur de la Guadeloupe à l'amiral Robert du 4 décembre 1940.

41 ADG. INC 140.

42 « À partir de décembre 1940, les États-Unis consentent à ouvrir un crédit mensuel de $600000 \$$ au profit des Antilles et de la Guyane françaises. Mais le contrôle du commerce extérieur des ÉtatsUnis, par le moyen de licences d'exportation, limite l'approvisionnement de ces colonies. » Page 73. Roger JAFFRAY, coordonnateur, Fédération Nationale du Mérite Maritime et de la Médaille d'honneur des marins. «Les transports maritimes aux Antilles et en Guyane françaises depuis 1930. L'Harmattan, 2009.

43 ADG. INC 135. Lettre du gouverneur de la Guadeloupe à l'amiral Robert du 6 novembre 1940. Série M.
} 
pour obtenir les dollars nécessaires mais à l'Attaché financier ${ }^{44}$ et ferait parvenir à ce dernier les commandes individuelles auxquelles serait joint un duplicata des licences d'importation accordées à la Guadeloupe dans les limites du plan de ravitaillement. »

Il faut ici faire un point sur la situation monétaire de la colonie de la Guadeloupe. Elle dispose localement d'un système monétaire distinct de la France depuis 1853, date de la création de la Banque de la Guadeloupe. Cette institution fonctionna jusqu'en 1944. Elle disposait du droit d'émission du franc guadeloupéen valant le même poids en or ou en argent que le franc français. Alain Buffon explique que « en pratique, le moyen de règlement à l'extérieur est la lettre de change ou traite sur la métropole. L'offre de traites représente la contrepartie financière de toutes transactions qui rendent le pays créancier; elle dépend donc du volume des exportations et également du prix du sucre sur le marché ${ }^{45}$.»

Les archives relatives au fonctionnement de la Mission d'Achat à New York, comme plusieurs autres documents originaux, manquent pour que nous puissions conclure sur le fonctionnement de cette mission et si les agents qui y ont été employés pendant la période vichyste de la Guadeloupe ont continué leur travail, lorsque la Guadeloupe s'est ralliée à la France libre. Une partie de ces archives a été détruite par un incendie en 1955 et les documents sauvés des flammes n'ont pas encore fait l'objet d'un classement exhaustif par le service des archives départementales de la Guadeloupe.

Cependant, la pénurie en morue observée dès 1940 reposerait sur les effets conjugués de la suspension des liaisons commerciales nationales, du manque de ressources financières de la colonie de la Guadeloupe et du manque de locaux pour le stockage des quantités de morue pouvant être acheminées depuis Saint-Pierre et Miquelon. Les 250 tonnes de morue importées à la suite du départ du chalutier Le Volontaire en juillet 1940 ne pouvaient être ni stockées ni conservées par manque d'infrastructure ${ }^{46}$.

Le débarquement des Forces françaises libres le 24 décembre 1941 à Saint-Pierre et Miquelon a eu des conséquences sur les rapports commerciaux entre l'archipel de l'Atlantique Nord et la Guadeloupe. Il faudra trouver un autre fournisseur. La

\footnotetext{
44 Voici les missions de l'attaché telles que décrites dans le rapport du capitaine Jacques Humbert : « 1 : accorder les crédits en dollars, et permettre les transferts de fonds. 2 : transmettre les commandes aux fournisseurs indiqués sur les documents par l'intermédiaire de la Banque Officielle à New York. 3 : faciliter l'octroi des licences d'exportation du Gouvernement américain. Il exercerait ainsi un rôle de coordination, de contrôle des fonds et d'intervention auprès des autorités des États-Unis. Pour faciliter d'ailleurs sa tâche, les commandes seraient groupées à la Guadeloupe par les Organismes Officiels du Commerce et de l'Industrie. Une seule commande serait établie pour un même article. Les intérêts de la population locale seraient entièrement sauvegardés et même protégés. » ADG, INC $163 / 4$.

45 BUFFON, Alain (2002). Les vielles banques coloniales d'émission : la Banque de la Guadeloupe et la Banque de la Martinique. Bulletin de la Société d'Histoire de la Guadeloupe, (132), page 56.

46 ADG. INC 135. Lettre du gouverneur de la Guadeloupe au Haut-Commissaire Robert du 7 août 1940. Série M.
} 
Mission d'Achats qui à sa création ne concernait pas les approvisionnements en morue servira d'intermédiaire et apparaîtra comme le seul interlocuteur pour les achats de morue pour l'administration française en Guadeloupe.

\section{La province de Terre-Neuve et Labrador fournisseur principal durant l'administration par le Comité Français de la Libération Nationale (CFLN).}

Les six derniers mois de l'administration de Vichy sont marqués par les manques. Cependant, grâce à la lecture des mercuriales de la douane publiées mensuellement dans le Journal Officiel de la Guadeloupe (JOG), nous avons les cours officiels des marchandises vendues, ainsi que parfois l'origine de celles-ci. L'examen des mercuriales de l'année 1943 montre quelques denrées alimentaires dont beaucoup sont d'origine américaine : les États-Unis fournissent du bœuf salé, ou la farine de froment; la République Dominicaine expédie du riz et du maïs, et le Chili des lentilles ${ }^{47}$. La morue est le seul poisson importé encore proposé à la vente, mais sans précision de l'origine de celle-ci. Comment Terre-Neuve et Labrador est-elle devenue fournisseur de la colonie?

\section{Une tentative de substitution de la morue dans l'alimentation guadeloupéenne : la politique de l'effort guadeloupéen}

Les quelques caisses de morue livrées depuis Saint-Pierre et Miquelon ne suffissent pas à écarter les craintes de la population. Le professeur de lettres, Lénis Blanche, le rapporte dans un témoignage $\operatorname{tardif}^{48}$ : «Ayant écrit au gouverneur que la pénurie de morue engendrerait une « faim d'azote ${ }^{49}$ » préjudiciable à la santé publique, et que l'arrêt de l'importation du « bois du Nord » poserait bientôt des problèmes, j'organisai en toute hâte " l'Exposition de la Mer et de la Forêt » à la Pointeà-Pitre. Il y eut ensuite " l'Exposition de l'Effort Guadeloupéen », qui montra au public des prodiges de créativité. L'effort en question permit d'échapper à la famine : nous pûmes même exporter vers la Martinique des vivres excédentaires. » Comment s'est organisée cette nouvelle politique?

La colonie dispose de plusieurs leviers : encourager la pêche pour augmenter la consommation de poisson frais, favoriser les techniques de conservation... Il faut encourager la population à consommer des produits locaux. C'est dans cette perspective que le gouverneur signe un décret en décembre 1940 pour organiser une exposition générale ${ }^{50}$.

Le gouverneur Sorin reprend une initiative de son prédécesseur qui avait rédigé une circulaire spécifique ${ }^{51}$ le 11 janvier 1940. Pour l'exposition générale de

\footnotetext{
47 JOG année 1943, 3K135.

48 Extrait du blogue «Le Scrutateur » d'Edouard Boulogne http://www.lescrutateur.com/ article-13030762.html. Lettre de Lénis Blanche à Edouard Boulogne du 31 mars 1979.

49 Ou manque de protéines.

50 Le programme officiel est paru au JOG du 11 janvier 1941. ADG, 3K133.

51 ADG. Circulaire du gouverneur de la Guadeloupe et dépendances à MM. Les Maires sur la
} 
février 1941, le gouverneur Sorin ne se contente pas l'organiser avec les services de l'état. Il demande également le concours de la population pour rassembler les objets nécessaires à l'exposition. La pêche est le premier secteur qui sera cité par le décret du 17 décembre $1940^{52}$ qui annonce et organise « une exposition générale des ressources, des techniques et des arts de la Guadeloupe du 8 au 11 février 1941. Selon ce même arrêté, le pavillon de la mer aura trois buts principaux. Il exposera : «1) les produits marins (animaux, végétaux et minéraux); 2) les moyens de capture, de ramassage et de conservation de ces produits; 3 ) les moyens propres à en développer la consommation. »

À cause du manque de morue, la colonie choisit le poisson frais comme produit de substitution. Or le commerce du poisson sévèrement encadré par l'arrêté $n^{\circ} 745$ du 16 avril $1940^{53}$ qui interdit « la sortie de poisson frais, sec, salé du territoire de la Guadeloupe et de ses dépendances (Saint Martin et Saint-Barthélemy exceptés) est provisoirement interdit sans autorisation du gouverneur. » En 1939, SaintBarthélemy comptait soixante-quinze embarcations de pêche pour deux cents vingt-cinq pêcheurs ${ }^{54}$. Une grande partie de leurs captures est salée avant d'être expédiée en Guadeloupe pour y être vendue.

Bien que les expositions aient mis en avant la pêche locale, on ne peut taire les difficultés des marins-pêcheurs. Ils manquent de matériels spécifiques (licences d'importation pour lignes de pêche). Ils manquent également de moyens financiers pour acheter des embarcations. C'est ainsi que la colonie institue un crédit maritime par l'arrêté $n^{\circ} 1132$ du gouverneur Sorin le 21 mai $1941^{55}$ pour mettre en place « un système d'avances aux marins-pêcheurs, inscrits maritimes de la Guadeloupe et dépendances en vue de faciliter le ravitaillement de la colonie en poisson » (art. 1). Mais ce système est également ouvert aux marins-pêcheurs retraités, à leurs veuves et aux orphelins jusqu'à leur majorité (art. 2). C'est la caisse locale du Crédit Agricole de Pointe-à-Pitre qui aura la gestion de ces prêts pour l'achat de matériels de pêche et d'embarcations (art. 8). Ces incitations financières et la valorisation du travail des marins-pêcheurs au travers des expositions ne peuvent masquer les problèmes de conservation du poisson frais local.

Le sel est un agent de conservation alimentaire largement employé en Europe mais peu utilisé par les Guadeloupéens. En Grande-Terre, il existe des salines inexploitées, sans perspectives d'être remises en production. Cependant, une tentative de fabrication de sel par l'usine sucrière de Marquisat (Capesterre-BelleEau, île de Basse-Terre) avait bien été ordonnée mais abandonnée rapidement. Car le sel produit était vert en raison de l'oxydation des tubes en cuivre de l'usine! Il ne restait plus qu'à récolter le sel issu de l'eau de mer bouillie.

nécessité d'accroitre les ressources locales en matière d'agriculture, d'élevage et de pêche du 11 janvier 1940. JOG du 18 janvier 1940.3K132.

52 ADG, JOG du 26 décembre 1940.3K132.

53 ADG, JOG du 18 avril 1940, 3K132.

54 BRETA, Félix. Contribution à l'étude des poissons vénéneux aux Saintes. 1939. Page 12.

55 ADG, JOG du 24 mai 1941. Arrêté nº 1132. 
Il y aurait une possibilité d'importer du sel depuis Saint-Martin où du sel de bonne qualité est tiré des salines. En 1940, la production de sel saint-martinoise est estimée à 80000 barils (1 baril valant $115 \mathrm{kgs}) .{ }^{56}$ Cependant, une anecdote m'a été rapportée : le gouverneur Sorin avait décidé de réquisitionner le sel produit par Louis-Constant Fleming au cours d'une visite sur l'île. En plus d'être producteur de sel, M. Fleming (1898-1949) était maire de la partie française de Saint-Martin entre 1925 et 1949 ainsi que commerçant. Le gouverneur Sorin tenta de lui imposer un prix d'achat inférieur à celui demandé. Dès que le gouverneur quitta SaintMartin, M. Fleming entreprit d'entreposer son sel dans la partie néerlandaise de l'île restée fidèle à la reine Whilemina, donc opposée à Vichy. Inutile de dire qu'au retour du gouverneur Sorin, il n'y avait plus de sel français disponible et que le sel néerlandais si proche ne lui serait pas vendu. L'absence d'un sel de qualité empêchera les Guadeloupéens à l'utiliser pour conserver leurs aliments.

Parallèlement, la réfrigération du poisson est limitée par la capacité de fabrication de barres de glace alimentaire dans les usines de Guadeloupe. Or cette glace nécessite des produits chimiques qui doivent être importés, donc tributaires des aléas du service du ravitaillement.

Grâce à l'arrêté n ${ }^{\circ} 1284$ portant sur la taxation du poisson frais du 9 juin $1941^{57}$, nous apprenons que : « la bonne qualité des poissons vidés frais ou frigorifiés de toutes catégories sera constatée par le vétérinaire de chacune des circonscriptions de Basse-Terre et de Pointe-à-Pitre. Les espèces reconnues nocives seront saisies pour être détruites, après avoir été rendues impropres à la consommation par leur immersion dans un mélange de formol ou d'acide phénique ». Or la liste des espèces de poissons visées par le même arrêté montre que dans les espèces de poissons réputés sains, il y a des espèces qui posent problèmes : le pagre à dent de chien (Lutjanus jocu), la carangue jaune (Caranx bartholomaei) et la bécune (Sphyraena barracuda $)^{58}$.

Malgré tous ces efforts, rien ne vient remplacer la morue, poisson de référence même si sa qualité durant le conflit est plus proche de la charpie. Les filets de morue de bonne qualité sont connus localement comme morue grand-banc mais les témoins de l'époque n'en n'ont pas le souvenir d'en avoir vus à l'achat ou même consommés durant la période de conflit. Confirmant ces informations, un témoignage d'un Guadeloupéen, M. Marcel Cyrille ${ }^{59}$ alors adolescent, m'a confié que la morue vendue à Capesterre-Belle-Eau par M. Voulzy, commerçant local, arrivait souvent en très mauvais état et qu'il devait plonger dans les barils pour tenter de trouver les plus gros morceaux de morue mais que la plupart du temps,

\footnotetext{
56 BLANCHE, Lénis. La pêche maritime en Guadeloupe. ADG INC 940.

57 ADG, JOG du 10 juin 1941. 3K133

58 Depuis, ces trois espèces de poissons sont interdites à la pêche et à la consommation en Guadeloupe, à Saint-Barthélemy et à Saint-Martin. Arrêté préfectoral (Guadeloupe) $n^{\circ}$ 2002-1249.

59 Pour la majorité des témoins le Tan Sorin est synonyme d'autosuffisance alimentaire dont ils tirent une très grande fierté. Très peu se souviennent du blocus à l'origine de la politique d'encouragement de la production locale.
} 
c'était de la charpie.

Cependant, qu'en est-il de l'ordre public que l'amiral Robert souhaitait avec force maintenir dans les colonies et qui l'avait conduit à s'intéresser aux questions de ravitaillement? Le paradoxe c'est que l'application en Guadeloupe de la politique du gouvernement de Vichy va conduire à l'emprisonnement des opposants politiques au Fort Napoléon (Terre-de-Haut), à la mise en place d'une réglementation sur les Juifs, aux exactions des marins d'origine européenne de la Jeanne d'Arc... et que le mouvement dit de la « dissidence » se développera grâce aux embarcations que vont « emprunter » les patriotes Guadeloupéens désireux de servir du côté du général de Gaule. Le 15 juillet 1943, le gouverneur Sorin est chassé du pouvoir par des représentants du CFLN et la population guadeloupéenne. Ce changement d'allégeance ne met pas un terme aux problèmes d'approvisionnement mais, désormais, la Guadeloupe est dans le même camp que ses principaux voisins. Comment faire pour organiser le ravitaillement en morue?

\section{L'arrivée de Terre-Neuve comme fournisseur possible pour l'administration de Vichy}

Les possibilités d'achat à l'étranger ne signifient pas que les entrepôts de la Guadeloupe reçoivent constamment des marchandises. En 1942, l'administration fait plus souvent face au manque. Mensuellement, l'administration fait paraître des mercuriales dans le $\mathrm{JOG}^{60}$ de la colonie qui permettent de disposer du prix légal des marchandises pour les professionnels et de calculer les droits et taxes afférents. Cependant, nous pouvons lire dans le JOG du 27 juin, la mercuriale des prix du mois de novembre 1941. Dans celui du 11 juillet, celle de janvier 1942 et dans celle du 18 juillet, celle de février 1942. La mercuriale publiée le 27 juin 1942 affiche les cours légaux de la farine et des lentilles d'origine états-unienne, du riz ou des pommes de terre, mais pas de la morue qui est manquante. Celle du 11 juillet indique le cours légal de la morue et des sardines marinées ou à l'huile tout comme celle du 18 juillet. Il semblerait que la colonie ait décidé de mettre en vente le plus tard possible les marchandises qu'elle reçoit, y compris lorsqu'il s'agit de l'alimentation. Elle administre la raréfaction des marchandises tout en stockant ces dernières dans les entrepôts sous douanes de la colonie et en ne les rendant pas disponible à l'achat pour la population.

Dans une lettre du 6 juillet 1942 au chef du service des Échanges commerciaux et du Ravitaillement, à Basse-Terre, M. Camille Bune ${ }^{61}$ propose à la colonie d'acheter de la morue à Terre-Neuve. Un autre document émis cette fois par le Bureau de ravitaillement en date du 12 juillet 1942, faisant un point sur la situation au 11 juillet 1942, indique que Camille Bunel est un commerçant pointois : «faisant preuve de bonne volonté (nous ne pouvons pas dire autant de tous les

\footnotetext{
60 ADG, JOG année 1942, 3K134.

${ }^{61}$ ADG. INC 135. Série M.
} 
commerçants de Pointe-à-Pitre $)^{62}$. » Est-ce le même Camille Bunel qui fut membre de la Chambre de Commerce de Pointe-à-Pitre dans les années Trente?

Dans sa lettre du 6 juillet, Camille Bunel se présente comme l'agent de la maison G.E.O.M Barr (sic) de Terre-Neuve. Cette dernière pourra expédier de la morue depuis Terre-Neuve par voilier tout comme il le fait pour le compte de la Martinique, explique-t-il. Le dossier que nous avons pu consulter ne comporte pas d'autres précisions à propos de la maison G.E.O.M Barr. Nous ne savons pas comment cette société a été mise en relation avec la Martinique, ni depuis combien de temps, elle est devenue fournisseur de cette colonie. Cependant, le document du Bureau du ravitaillement en date du 12 juillet 1942, cité plus haut, confirme que ces liens sont connus de l'administration de la Guadeloupe puisqu'elle écrit que « ...la maison B.A.R.R est une maison de confiance... »

Nous ne pouvons confirmer que « l'expédition Bunel », comme le nomme les documents, ait été un succès. Il faut dire qu'outre les $60000 \$$ de morue, le Bureau de ravitaillement propose au gouverneur de la Guadeloupe un arbitrage car il doit également payer $11000 \$$ de farine de manioc et $13000 \$$ d'allumettes et de savons importés de Cuba, alors que la Guadeloupe ne dispose plus que de $23000 \$^{63}$. Bien sûr, il y a la possibilité de faire un crédit auprès de la maison G.E.O.M Barr, comme 1'explique Camille Bunel, mais cela se ferait au détriment d'autres marchandises payées également en dollars US.

Il est regrettable que d'autres bulletins de situation du Bureau du ravitaillement ne nous soient pas parvenus. Ils nous auraient éclairé sur les fournisseurs et la façon dont l'administration a fait ses arbitrages. Cependant, l'unique bulletin du 6 juillet 1942 indique que M. Thomasset, basé à Saint-Domingue, agit directement pour acheter du riz dans l'île et du savon et des allumettes à Cuba. C'est également le même Thomasset qui n'hésite pas à demander le soutien des forces de l'Axe. Ainsi, nous pouvons lire que : «M. Thomasset a demandé à M. Poirier, lors de son départ pour la Martinique, de tacher d'obtenir de l'Axe que les bateaux et goélettes de Saint-Domingue travaillant exclusivement avec les Antilles françaises ne soient pas coulés. Il est urgent de connaître les résultats de cette demande. » Nous pouvons conclure qu'alors même que la Guadeloupe s'approvisionne auprès des États-Unis et de son allié dominicain, les liens ne sont pas totalement rompus avec l'état-major de l'Axe à moins qu'il ne s'agisse d'un fantasme de M. Thomasset sur les relations réelles ou supposées de la Martinique avec l'Axe. C'est la Martinique qui a l'autorité sur le volet défense, d'où l'ignorance de la Guadeloupe concernant le mouvement des bateaux et sous-marins de l'Axe dans les eaux caribéennes.

Dans son ouvrage sur «Les Transports maritimes aux Antilles et en Guyane françaises depuis $1930^{64} »$, la Fédération nationale du Mérite maritime et de la

62 Id.

63 Id. Note sur la situation au 11 juillet 1942.

64 La Fédération nationale du Mérite maritime et de la Médaille d'honneur des marins coordonnée par Roger Jaffray. Les transports maritimes aux Antilles et en Guyane françaises depuis 1930. l'Harmattan, 2009. Page 94. 
Médaille d'honneur des marins estime que le commerce extérieur de la Guadeloupe, entre 1940 et 1943, passe de 150000 tonnes à 28000 tonnes. À la même époque et selon la même source, le commerce extérieur de la Martinique est réduit de 275000 tonnes à 75000 tonnes.

\section{Les défis de la France libre}

Le 14 juillet 1943, la Martinique se rallie aux Forces françaises libres et le lendemain la Guadeloupe fait de même. Toutes deux passent sous le commandement d'Henri Hoppenot ${ }^{65}$, ministre plénipotentiaire du CLNF aux Antilles françaises. Ce ralliement entraîne un large mouvement de changement de postes à la tête de la colonie, tant sur le plan militaire que civil. Le ministre Hoppenot nomme successivement Georges Poirier ${ }^{66}$, puis Maurice Bertaut ${ }^{67}$ au poste de gouverneur de la Guadeloupe.

Lorsque le gouverneur Poirier arrive au pouvoir, la colonie vient de vivre six mois très difficiles à cause des défaillances sur les produits importés. La morue qu'elle soit « morue GB » (grand bancs) ou morue PP (petit poisson) est le plus souvent manquante depuis le mois de janvier, seul avril fait exception ${ }^{68}$. La population se tourne vers le poisson pêché localement. Dans un arrêté du 28 juillet 1943 relatif à l'achat et à la vente du poisson frais et conservé en frigorifique pour la Guadeloupe et ses dépendances ${ }^{69}$, le gouverneur Poirier fixe le prix maximum de vente des poissons et du lambi (Strombus gigas) pour empêcher une flambée des prix. Mais la colonie est toujours en demande de morue et c'est à un nouvel opérateur de prendre le relais de la mission d'Achat : la société « French Colonial Supply Mission » basée à New York. À partir du ralliement de la Guadeloupe à la France libre, le ravitaillement de la Guadeloupe est toujours problématique : il n'y a pas de morue d'août à novembre comme le montrent les mercuriales des six derniers mois de $1943^{70}$.

Les documents conservés en Guadeloupe permettent de comprendre le nouveau mécanisme de ravitaillement. La colonie télégraphie une commande par produit à la French Colonial Supply Mission, suivant un modèle que le gouverneur Sorin

\footnotetext{
65 HOPPENOT, Henri Étienne (25 octobre 1891 - 10 août 1977, Paris, Île de France). Il se rallie aux Forces françaises libres en 1943 après avoir été nommé ministre plénipotentiaire à Montevideo (Uruguay) par le gouvernement de Vichy. Il finit par prendre le parti du général De Gaulle qui le nomme, en juin 1943, délégué du CFLN aux États-Unis d'Amérique, puis ministre plénipotentiaire du CFLN aux Antilles françaises.

${ }_{66}$ POIRIER, Georges Émile Albert (4 septembre 1894 Vagney, Vosges-?), ancien secrétaire général du gouverneur Constant Sorin, en Guadeloupe. Rallie la France libre. Gouverneur de la Guadeloupe du 15 juillet 1943 au 29 août 1943.

67 BERTAUT, Pierre Maurice Eugène François Stéphane (10 octobre 1900 Le Tampon Saint-Pierre, La Réunion- 23 décembre 1994, Paris 14 ${ }^{\text {ème }}$, Île de France). Fonctionnaire, nommé gouverneur de la Guadeloupe du 29 août 1943 à 1946.

68 ADG, JOG 24 avril 1943. 3K135.

69 Id. 31 juillet 1943.

70 ADG 3K 135. JOG, année 1943.
} 
avait suggéré. C'est lui qui est chargé de trouver des fournisseurs et de les payer. La copie des ordres d'achat est envoyée à la Guadeloupe ${ }^{71}$. La lecture de ces ordres d'achat nous apprend que l'intermédiaire sur place fait le choix de la marchandise. Durant l'année 1944, la société « Puerto Rico Exporters, Limited » de Saint-Jean de Terre-Neuve apparaît comme représentant. Cette société agit également comme intermédiaire pour la Martinique. Entre les deux colonies françaises, le partage ne se fait pas de façon égalitaire. Dans le document «Agreement for the purchase of Newfoundland salt codfish - 1943 ${ }^{72}$, , les ratios sont clairement indiqués : $60 \%$ pour la Martinique et $40 \%$ pour la Guadeloupe pour chaque expédition. Les deux colonies françaises vont recevoir de la morue, mais elle ne correspond pas à la nomenclature locale faite de «morue PP » et de «morue GB ». Aucun document nous indique la façon dont l'administration guadeloupéenne va faire coïncider leur nomenclature à la nomenclature canadienne.

Mais ces difficultés ne vont pas remettre en cause les approvisionnements via la French Colonial Supply Mission, d'autant que cette administration de la France libre est la seule en charge de l'approvisionnement pour les territoires français de l'Afrique occidentale française, l'Afrique équatoriale française, le Cameroun, Madagascar, les Antilles et la Guyane française, les possessions du Pacifique et Saint-Pierre et Miquelon ${ }^{73}$.

Une fois la marchandise trouvée à Terre-Neuve, il faut des moyens maritimes pour l'acheminer jusqu'en Guadeloupe de façon à y être vendue. Or il y a des difficultés dans le traitement des commandes qui passent par plusieurs administrations, soit un secrétariat permanent ${ }^{74}$, puis la Mission de Liaison Économique des Antilles (M.L.É.A.) et enfin par la Mission des Colonies. Dans son rapport de fin de mission $^{75}$, le capitaine Jacques Humbert, chef de la M.L.É.A au 30 décembre 1944, explique que plus de 500 commandes transmisses à la M.L.É.A ont été divisées en plus de 1100 dossiers qui seront encore divisés, pour raison de service. Un autre problème est soulevé par le chef de la M.L.É.A, celui du suivi des commandes. En effet, ce dernier était incapable de faire la part des commandes livrées en totalité, de celles partiellement livrées ou de celles qui avaient été annulées. Il en résulte que nul ne peut mesurer l'efficacité du service rendu par la M.L.É.A pour l'approvisionnement de chaque colonie. Une dernière difficulté est d'assurer le règlement de chaque commande. C'est à la M.L.É.A qu'il convient d'affecter le bon type de règlement suivant la nature des produits commandés.

À chaque commande est annexée un document fixant les conditions relatives au paiement des cargaisons de morue venues de Terre-Neuve. La Banque de la

\footnotetext{
71 ADG. Série continue, carton 434.

72 Id.

73 ADG. INC 163/4. Rapport de fin de mission du capitaine Jacques Humbert, chef de la M.L.É.A. Série M.

74 Id.

75 Id. Page 8.
} 
Guadeloupe, créée par la loi du 11 juillet $1851^{76}$, est une banque d'escompte et d'émission distincte de la Banque de France qui va voir son privilège d'émission constamment prolongé par l'administration centrale française. Du fait de sa nature particulière, la Banque de la Guadeloupe va pouvoir être un opérateur financier pour le paiement des commandes par la M.L.É.A. Dans ce but, elle va poursuivre sa collaboration avec la French American Banking Corporation ${ }^{77}$. Dans les archives internes de la banque, nous pouvons lire une minute ${ }^{78}$, en date du 27 mars 1940, qui indique que la Banque de la Guadeloupe, comme celle de la Martinique, y disposait d'un dépôt d'une valeur de 50000 \$.

Les mouvements bancaires des Antilles françaises vont augmenter rapidement. Ils atteindront $1000000 \$$ par mois pour financer les achats de marchandises dès 1942. Or nous sommes bien loin des 100000 dollars en devises disponibles pour les achats durant l'administration Sorin. Ce montant de 1000000 \$ va se maintenir et en 1943, une minute ${ }^{79}$ de la banque indique qu'elle dispose sur ses comptes américains suffisamment de fonds pour couvrir les besoins des Antilles françaises pour six mois d'activités, couvrant à la fois les achats de marchandises et des fonds disponibles. Elle ajoute que si les Antilles françaises réussissent leur projet de production de sucre et de rhum, elles deviendraient « virtuellement autonome ». Ce qui est surprenant, c'est que malgré les montants importants évoqués dans les notes internes de la French American Banking Corporation, les populations des Antilles françaises, et particulièrement de la Guadeloupe, ont subi de très nombreuses ruptures d'approvisionnement.

Les lettres de crédit établies par la French American Banking Corporation en faveur de la société Puerto Rico Exporters ont une validité de 90 jours. Cependant, la French American Banking Corporation n'étant pas présente sur place, elle charge son représentant local à Saint-Pierre et Miquelon, la Royal Bank of Canada ${ }^{80}$, de les confirmer et d'assurer en sus le contrôle des documents administratifs obligatoires. Ces lettres de crédit sont révocables en cas de défaillance du vendeur. Malgré toutes ces mesures, en Guadeloupe il y a toujours des difficultés à la mise en vente de la morue et l'administration va choisir de mettre en place des rations.

Le rationnement de la morue mais aussi de plusieurs produits de première nécessité conduit l'administration de Vichy à mettre en place des cartes de

\footnotetext{
76 BUFFON, Alain. Monnaie et crédit en économie coloniale : contribution à l'histoire économique de la Guadeloupe 1635-1919. Société d'histoire de la Guadeloupe 1979. La France crée à cette occasion trois banques : celle de la Guadeloupe, de la Martinique et de La Réunion (page 150).

77 Banque franco-américaine créée en 1919, détenue à 50\% par le Comptoir d'Escompte de Mulhouse et le Comptoir National d'Escompte de Paris (actuellement, BNP-Paribas). Renseignements complémentaires, page 175. WILKINS, Mira. The History of Foreign Investment in the United States, 1914-1945 (Cambridge MA: Harvard University Press, 2004).

78 Archives historiques de la BNP-Paribas, dossier 73 AH 1232.

79 Id. Regular meeting of the board of directors of the French American Banking Corporation, January 13, 1943 10:30 A.M.

${ }_{80}$ C'est le nom commercial choisi pour ses succursales françaises de Saint-Pierre et Miquelon et la Guadeloupe. Cette banque a disposé d'une succursale en Guadeloupe en 1919 et l'annonce de sa cessation d'activité au 30 juin 1940 est publiée au JOG.
} 
rationnement conformément à l'arrêté local $\mathrm{n}^{\circ} 1591$ du 15 juillet $1942^{81}$. Cette politique est poursuivie par l'administration de la France libre. Dans le JOG du 9 octobre $1943^{82}$, le gouverneur détaille à nouveau le fonctionnement de ces cartes de rationnement et des sanctions encourues en cas d'infraction par les contrevenants dans ses « instructions générales relatives à la répartition et à la mise en vente des denrées, produits et objets de première nécessité. » La morue, denrée de première nécessité, figure dans l'annexe de l'arrêté. Bien que la population soit demandeuse de morue, la colonie ne peux pas mettre en vente toutes les cargaisons sans subir un contrôle sanitaire.

La morue contrôlée au départ des ports de Terre-Neuve sera à nouveau contrôlée à son arrivée en Guadeloupe par le service vétérinaire des colonies ${ }^{83}$. L'arrêté $\mathrm{n}^{\circ} 1095$ du 6 mai 1942 relatif à la fabrication et à la mise en vente du poisson précise dans son article 9: «À l'arrivée en Guadeloupe, le poisson salé, séché ou fumé est soumis à un examen de la part du médecin ou d'un vétérinaire désigné par le gouverneur et du chef du service des Échanges Commerciaux ou de son représentant. Le poisson refusé par eux est obligatoirement incinéré ou employé comme engrais. Toutefois, si le refus est basé seulement sur une salaison, un séchage, un fumage insuffisant, ils peuvent autoriser la mise en consommation immédiate dans le port de débarquement. » Rien ne nous permet de conclure sur le volume des refus administratifs, les documents que nous avons pu consulter étant très lacunaires.

Bien que l'administration de la France libre apparaisse comme le continuateur des solutions de ravitaillement mises en place par l'administration de Vichy, c'est à elle que revient le défi d'organiser l'après-guerre et la fin des approvisionnements de la colonie par voie administrative. C'est ainsi que le 24 mars 1944, Jean-Paul Lévi, chef de la Mission des colonies aux États-Unis mais également membre du conseil français des approvisionnements en Afrique du Nord et James Farris, fonctionnaire à la section économique du Département d'État des États-Unis arrivent en Martinique. C'est dans cette colonie que se tient une réunion avec les fonctionnaires qualifiés des Antilles et de la Guyane française. Ils viennent préparer la réouverture à la commande privée. Cependant, le communiqué du gouverneur Bertaut précise que la morue ne sera pas concernée par ces changements à venir tout comme la farine, les engrais, le charbon, les pneumatiques, les camionautomobiles mais également les produits « cédés par une agence gouvernementale des États-Unis » et les produits vendus par la République d'Argentine.

L'administration de la France libre a poursuivi les efforts de l'administration de Vichy pour assurer les approvisionnements de la Guadeloupe. Pour la morue,

\footnotetext{
81 ADG JOG 22 juillet 3K134.

82 ADG 3K135.

83 Ce décret du 24 mars 1939 portant organisation du cadre général des services vétérinaires est cité dans un rapport à M. le gouverneur $\mathrm{n}^{\circ} 2938 \mathrm{EC}$ : arrivage de poisson salé par « INES II » 20 mai 1943. ADG INC 135. Série M.
} 
si l'administration sait que Terre-Neuve a dû remplacer Saint-Pierre et Miquelon comme fournisseur, rien ne nous laisse penser que la population ait su que la morue n'était plus française. Car l'administration n'a pas toujours utilisé la nomenclature «morue G.B » et « morue P.P ».

Nous avons esquissé le circuit financier permettant de mieux découvrir le rôle de Puerto Rico Exporters Limited, de la Banque de la Guadeloupe et de la French American Banking Corporation. Ce faisant nous avons mis en lumière le montant des transitions financières passées par cette dernière et en faisant le rapprochement avec les montants des achats en devises déclarés au début du conflit, nous nous posons la question de l'utilisation des fonds pour le seul profit du ravitaillement de la colonie.

\section{Conclusion}

Entre 1939 et 1945, la Guadeloupe, indépendamment des influences politiques, a su exploiter au maximum les particularités de son statut de colonie pour faire face aux problèmes d'approvisionnement pendant toute la durée du conflit, que ce soit sous l'administration de Vichy ou de la France libre et assurer l'arrivée dans les assiettes guadeloupéennes de l'incontournable morue.

La première mesure a été de permettre à l'administration de se substituer au commerce privé pour assurer la fourniture des approvisionnements de l'archipel, grâce à la prise de l'arrêté 1970 du 28 décembre 1940 établissant un monopole d'importation de la morue française et étrangère qu'il réserve au service du Ravitaillement.

Les différentes administrations françaises ont tenté de maintenir les réseaux d'approvisionnement en morue en tentant de favoriser la morue française grâce à Saint-Pierre et Miquelon. Ces approvisionnements ont été possibles grâce à des négociations avec les États-Unis d'Amérique et à l'intervention de l'amiral Robert. Le Haut-Commissaire pour l'Atlantique Ouest, outrepassant les limites de son mandat, s'est impliqué dans les questions de ravitaillement et son action a permis des brèches dans la politique de blocus mis en place par le Royaume-Uni dès juin 1940. Cependant, consciente des difficultés liées aux approvisionnements extérieurs, l'administration a impulsé une réorganisation de la demande intérieure en proposant à la population comme alternative à la morue, le poisson frais local.

Cependant, les efforts constants de proposer une alternative à la morue, que ce soit par l'administration de Vichy ou de la France libre, ont conduit la colonie de la Guadeloupe à conserver des liens commerciaux avec Saint-Pierre et Miquelon et Terre-Neuve comme fournisseurs de morue. Le choix de l'un ou de l'autre étant tributaire des aléas de la guerre. La colonie de Saint-Pierre et Miquelon est d'abord choisie comme fournisseur puisqu'elle est un producteur historique de morue séchée mais également parce qu'elle est soumise à l'autorité militaire de l'amiral Robert, jusqu'au ralliement de Saint-Pierre et Miquelon aux Forces Françaises Libres en décembre 1941. À partir de 1942, c'est Terre-Neuve qui va principalement fournir l'archipel guadeloupéen en morue jusqu'à la fin du conflit. 
Or la morue, comme l'ensemble des produits importés, est payée grâce au travail des Guadeloupéens qui paient l'impôt et abondent le Trésor Public. C'est ainsi que la Guadeloupe, contre le modèle économique traditionnel colonie-métropole, ne semble plus dépendre économiquement de la France. Cette autonomie économique n'est pas une autonomie politique : le pouvoir central de la France libre ne laisse pas l'administration de la colonie aux Guadeloupéens, installant plutôt des gouverneurs qui lui sont loyaux à sa tête.

L'étude des approvisionnements morutiers a mis en lumière les différents leviers utilisés par la colonie de la Guadeloupe pour tenter de maintenir ou de proposer une alternative locale aux importations de morue. Or la morue n'a jamais déserté le quotidien des Guadeloupéens qui en consomment toujours, notamment sous la forme de ses célèbres « accras » de morue, de brandade et autres pâtés salés. Cependant le Comité Régional des Pêches de Guadeloupe, citant les chiffres du Système d'Information Halieutique de l'Ifremer ${ }^{84}$, estime la consommation en produits halieutiques (poissons et coquillages) à $14000 / 15000$ tonnes par an, soit $37 / \mathrm{kg}$ par habitant. La demande interne, pour sa part, est de 9000 tonnes/an, soit $60 \%$ de la demande. Ces chiffres montrent que la politique de retour à une pêche locale s'est alliée à un goût certain des Guadeloupéens pour les produits de la pêche. La pêche locale a bénéficié de l'impulsion donnée durant la Seconde Guerre mondiale pour augmenter dans les assiettes la part des produits halieutiques locaux, non pas en se substituant mais en complétant la demande pour l'incontournable morue.

${ }^{84}$ IFREMER : Institut Français de Recherche pour l'Exploitation de la Mer. 